\title{
Micro- and Submicrostructuring Thin Polymer Films with Two and Three-Beam Single Pulse Laser Interference Lithography
}

\author{
Ignacio Martín-Fabiani, ${ }^{*}{ }^{\dagger}$ Stephen Riedel, ${ }^{\ddagger}$ Daniel R. Rueda, ${ }^{\dagger}$ Jan Siegel, ${ }^{\S}$ Johannes Boneberg, ${ }^{\ddagger}$
} Tiberio A. Ezquerra, ${ }^{\dagger}$ and Aurora Nogales ${ }^{\dagger}$

${ }^{\dagger}$ Instituto de Estructura de la Materia (IEM-CSIC), Serrano 121, 28006 Madrid, Spain

${ }^{\ddagger}$ University of Konstanz, Fach 676, 78457 Konstanz, Germany

${ }^{\S}$ Instituto de Óptica (IO-CSIC), Serrano 121, 28006 Madrid, Spain
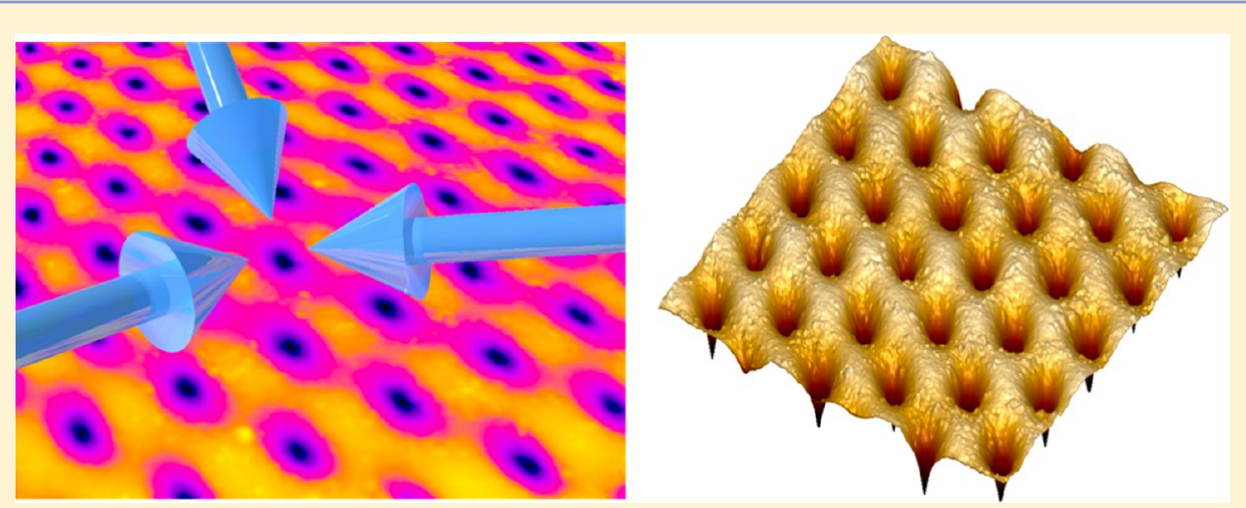

ABSTRACT: In this work we report the application of two and three-beam single pulse laser interference lithography to thin polymer films of poly(trimethylene terephthalate) (PTT). By irradiating the sample surface with temporary and spatially overlapped single pulses from two or three coherent beams and changing the angles of incidence, we have accomplished the fabrication of large-area polymer micro and submicrogratings as well as submicrometric cavities arranged in a hexagonal lattice. The characterization of the structures in real space by atomic force microscopy (AFM) and scanning electron microscopy (SEM) has allowed us to determine the formation mechanism of the microgratings to be based on different ablation regimes depending on the local fluence. Moreover, complementary characterization of the submicrometric cavities in reciprocal space by grazing incidence small-angle X-ray scattering (GISAXS) confirms the existence of large areas where two-dimensional order is present. The experiments presented in this work demonstrate the suitability of single pulse laser interference lithography for micro and submicrostructuring polymer films, opening up new possibilities for patterning and paving the way for potential applications where polymer structures are involved.

\section{INTRODUCTION}

Laser processing is one of the most used approaches to fabricate micro- and nanostructures. ${ }^{1}$ By irradiating with a high power laser beam, it is possible to directly write lines or cavities on the surface of the target material. ${ }^{2}$ Nevertheless, writing more than one of these features at a time requires the use of a mask. ${ }^{3}$ Laser interference lithography (LIL) allows overcoming this limitation and writing a whole array of structures at once. It is based on the selective modification of material by irradiation of the sample surface with two or more coherent beams. ${ }^{4}$ When two beams interfere, a periodic intensity pattern is generated yielding microor nanogratings, whose period depends on the angle formed between the beams. If three beams interfere (3-beam LIL) the pattern produced is hexagonal, thus forming cavities. ${ }^{5}$

Polymeric materials have become strong candidates in the past years for micro- and submicrostructuring, since potential applications might benefit from their excellent properties, i.e., mechanical flexibility, lightweight, enhanced durability, and low cost. Polymer nanostructures are being currently implemented in a wide variety of applications, such as biosensors, ${ }^{6}$ solar cells, ${ }^{7}$ and nonvolatile memories. ${ }^{8,9}$ In particular, poly(trimethylene terephthalate) (PTT) is a semicrystalline aromatic polyester whose outstanding mechanical and optical properties, together with its low glass transition temperature $T_{\mathrm{g}} \approx 44^{0} \mathrm{C},{ }^{10}$ make it an attractive material for the fiber industry ${ }^{19}$ as well as for optoelectronic $^{12}$ and nanophotonic ${ }^{13,14}$ applications. PTT has already shown interesting features when structured by several different methods such as laser-induced periodic surface structuration (LIPSS), ${ }^{15}$ nanoimprint lithography (NIL), ${ }^{16}$ and near-field patterning. ${ }^{17}$

Laser interference patterning of bulk polymers has led to applications such as substrates for increasing the efficiency of solar cells ${ }^{18}$ and diffraction gratings. ${ }^{19}$ However, only a couple of studies on a certain polymer blend can be found laser

Received: May 30, 2014

Revised: July 7, 2014

Published: July 8, 2014 


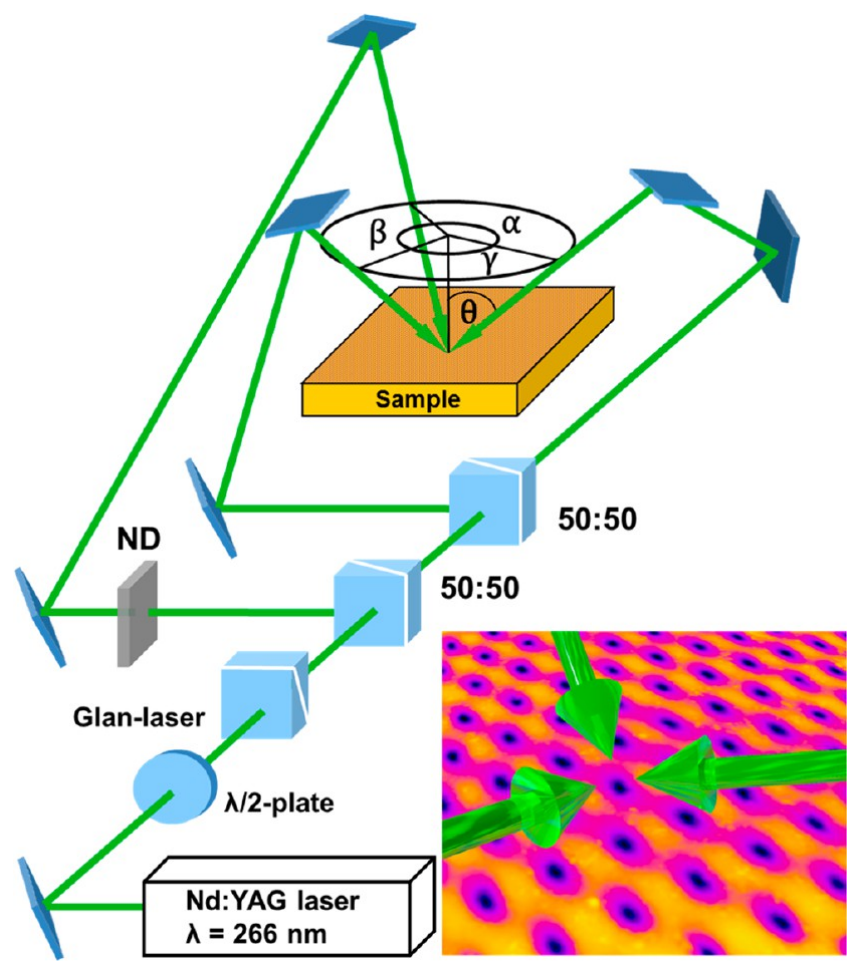

Figure 1. Optical setup used in the SPLIL experiments.

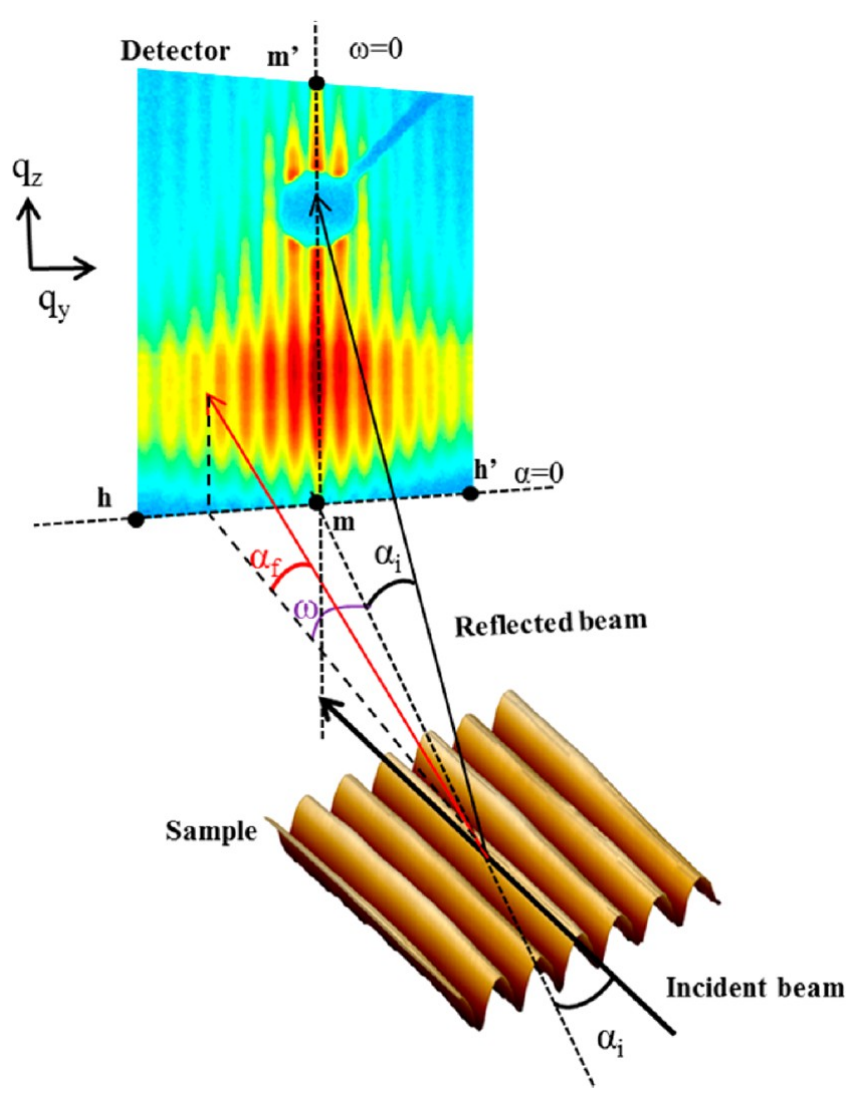

Figure 2. Schematic view of a GISAXS experiment. The scattering plane, containing both the direct and the specular beams, intersects the $2 \mathrm{D}$ detector along the meridian, $\mathrm{m} \_\mathrm{m}^{\prime}$ line, of the GISAXS pattern. The horizon, $\mathrm{h} \_\mathrm{h}^{\prime}$ line, is the intersection between the sample plane and the plane of the $2 \mathrm{D}$ detector, which are perpendicular to each other. Each point on the GISAXS pattern can be characterized by the exit angle, $\alpha_{\mathfrak{f}}$ and the out-of-scattering plane angle, $\omega$.
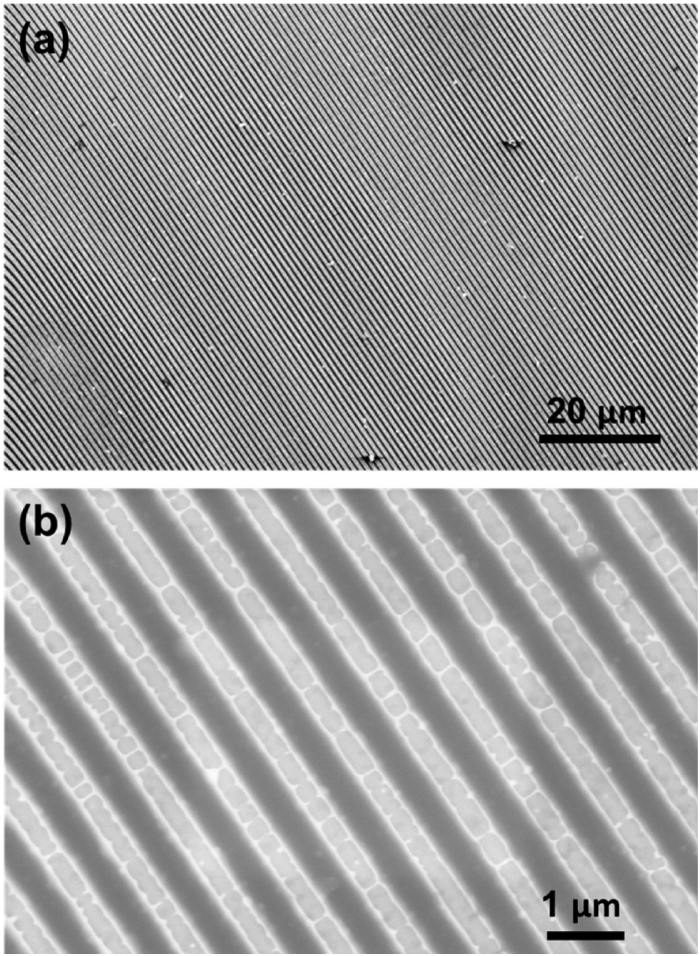

Figure 3. Microstructures generated in a PTT film by 2-beam SPLIL (angle of incidence $\theta=5^{0}$ ): (a) SEM micrograph of a large patterned area and (b) zoomed region.

interference patterning applied to polymer thin films, ${ }^{20,21}$ and the ablation mechanism involved in the structuring process is still under discussion. ${ }^{22}$ Moreover, the structures fabricated up to now using this lithographic technique are mainly micrometric, ${ }^{23,24}$ and submicrostructuration has been scarcely reported in this field for polymers. In this work we report the application of two and three-beam single pulse laser interference lithography (3-beam SPLIL) to micro- and submicrostructure thin polymer films of PTT, emphasizing the formation mechanism and the assessment of the resulting structures in real and reciprocal space.

\section{EXPERIMENTAL SECTION}

PTT was synthesized by polycondensation as previously described, ${ }^{25}$ yielding a molecular weight of $M_{\mathrm{n}}=31294 \mathrm{~g} / \mathrm{mol}$ and a polydispersity of $M_{\mathrm{w}} / M_{\mathrm{n}}=2.22$, as determined by size exclusion chromatography (SEC). PTT is a semicrystalline polymer, with a melting temperature of $T_{\mathrm{m}}=$ $229^{\circ} \mathrm{C}$ and a glass transition temperature of $T_{\mathrm{g}}=44^{\circ} \mathrm{C}$, as determined by calorimetry. Polymer thin films were prepared by spin-coating on silicon wafers (100) (Wafer World, Inc.) polished on one side. The wafers were previously cleaned with acetone and isopropanol. PTT was solved in trifluoroacetic acid (Sigma-Aldrich, reagent grade $\geq 98 \%$ ) with a concentration of $20 \mathrm{~g} / \mathrm{L}$. A fixed amount of $0.1 \mathrm{~mL}$ of polymer solution was instantly dropped by a syringe on a square (typically $2 \times 2 \mathrm{~cm}^{2}$ ) silicon substrate placed in the center of a rotating metallic horizontal plate. A rotation speed of $2380 \mathrm{rpm}$ was maintained for $30 \mathrm{~s}$. Spin-coated polymer films with a thickness of about $157 \pm 24 \mathrm{~nm}$ and extremely flat surface (mean surface roughness Ra $\leq 1 \mathrm{~nm}$ ), as measured by atomic force microscopy (AFM), are typically obtained under these conditions.

For the SPLIL experiments, we used nanosecond laser pulses from an injection-seeded Nd:YAG laser (Continuum Powerlite 9010, $\lambda=266$ $\mathrm{nm}, \tau=10 \mathrm{~ns}$ with a spot size of $5 \mathrm{~mm}$ on the sample surface). This laser wavelength was chosen in order to fall into the absorption band of PTT, with a linear absorption coefficient $\alpha_{266 \mathrm{~nm}}=25997 \mathrm{~cm}^{-1}$. The laser pulse with Gaussian beam profile is split into two or three beams of equal intensity, as in Figure 1. For two-beam experiments, $\alpha=\beta=180^{\circ}$, whereas for three beams, $\alpha \approx \beta \approx \gamma$. These beams are then recombined at 

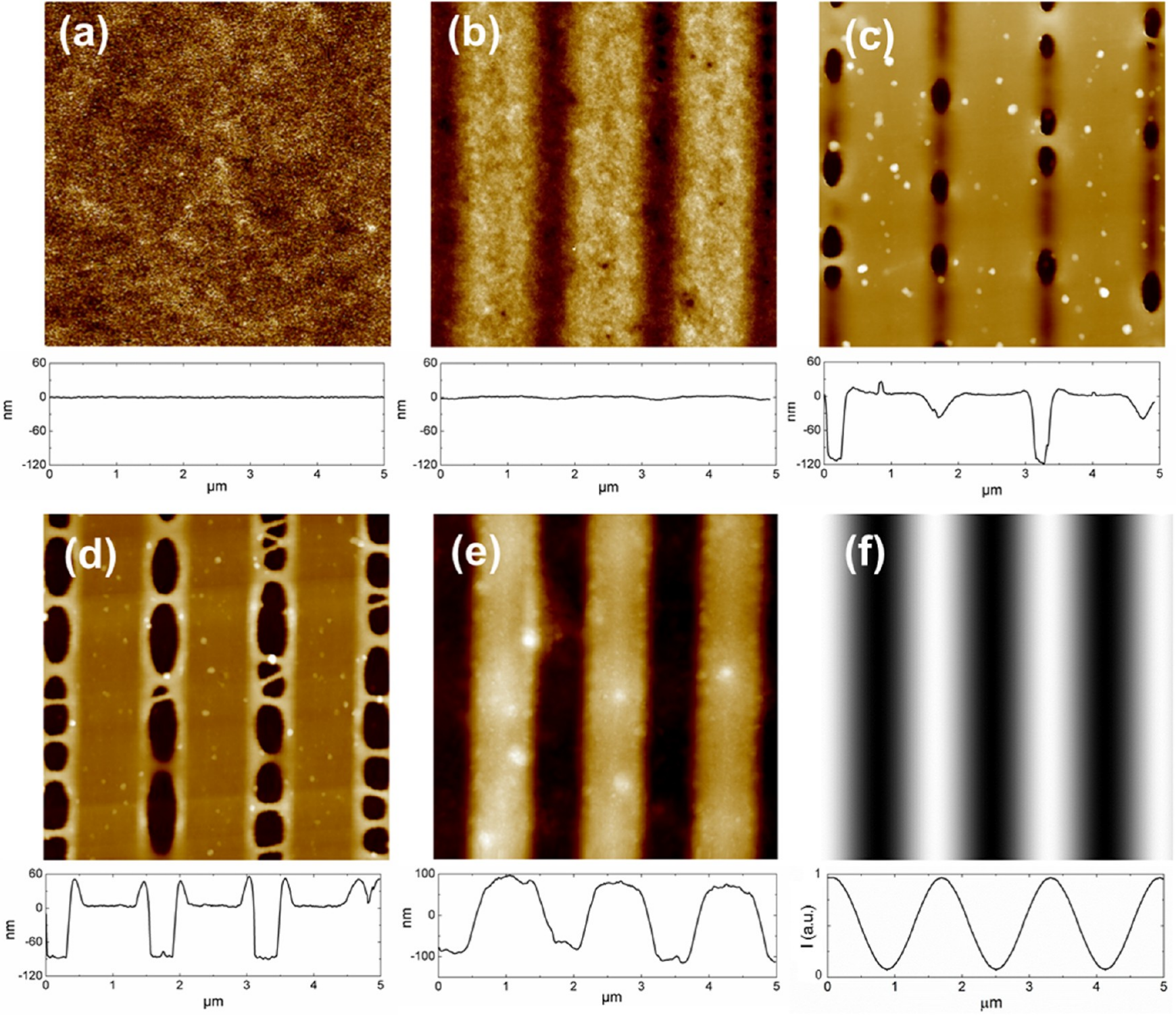

Figure 4. AFM topography images $\left(5 \times 5 \mu \mathrm{m}^{2}\right)$ of gratings on a PTT thin film fabricated by 2-beam SPLIL (angle of incidence $\left.\theta_{1}=8^{0}\right)$ taken at different areas of the sample: (a) outside the irradiated area and at four different regions inside the irradiated area in descending order of the distance to the beam center: (b) $3.2 \mathrm{~mm}$, (c) $2.8 \mathrm{~mm}$, (d) $2.4 \mathrm{~mm}$ and (e) $2 \mathrm{~mm}$. (f) shows the theoretical interference profile considering the geometry of the experiment. Below, profiles along a $5 \mu \mathrm{m}$ line perpendicular to the main axis of the structures.

the surface of the polymer thin film under a certain angle of incidence $\theta$, which defines the period of the interference pattern that will be produced: $\Lambda=\lambda / 2 \sin \theta$. After single exposure with typical laser fluences between $100-300 \mathrm{~mJ} / \mathrm{cm}^{2}$, a periodic pattern appears at the sample surface. These patterns are observed by the appearance of diffraction effects from a test laser beam that impinges on the structures.

After irradiation, the structures were characterized using scanning electron microcopy (SEM, CrossBeam 1540XB, Zeiss) and AFM (Nanoscope V, Bruker) in tapping mode. AFM images were analyzed using the software Nanoscope Analysis 1.40. Grazing incidence smallangle X-ray scattering (GISAXS) experiments were carried out in order to assess the structural order of the as-fabricated structures. They were conducted using the facilities of the BW4 beamline at the Deutsches Elektronen Synchrotron (DESY), Hamburg, Germany. ${ }^{26}$ A scheme of the experimental setup for GISAXS is shown in Figure 2. The information can be interpreted in terms of two orthogonal scattering vectors $q_{z}=(2 \pi / \lambda)\left(\sin \alpha_{\mathrm{i}}+\sin \alpha_{\mathrm{f}}\right)$ and $q_{y}=(2 \pi / \lambda) \sin \omega \cos \alpha_{\mathrm{f}}$, which provide information about structural correlations perpendicular and parallel to the film plane, respectively. ${ }^{27,28}$ Lateral correlation between scattering objects on the film surface can induce some scattered intensity appearing out of the meridian (line $\mathrm{m}_{-} \mathrm{m}^{\prime}$ in Figure 2). An incident angle of $\alpha_{\mathrm{i}}=0.4^{\circ}$ was chosen, and an $\overline{\mathrm{X}}$-ray wavelength of $\lambda=0.138 \mathrm{~nm}$, with a beam size $(H \times V)$ of $40 \times 20 \mu \mathrm{m}^{2}$, was used in our experiments. Scattered intensity was recorded by a Mar CCD detector of $2048 \times 2048$ pixels with a resolution of $172 \mu \mathrm{m}$ per pixel, and a sample-to-detector distance of $2.32 \mathrm{~m}$.

\section{RESULTS AND DISCUSSION}

In the first set of experiments, two-beam single pulse laser interference was applied to PTT thin films to obtain micro- and nanostructures with different periods, by changing the angles of incidence of the laser beam on its surface. For small incident angles $\left(\theta=5-8^{0}\right)$, the structures generated are microgratings, over an extensive area (see Figure 3a), expanding about half of the beam size $(2.5 \mathrm{~mm})$, with a period close to one micron (see Figure $3 \mathrm{~b}$ ). The laser interference fringes have been transferred into the polymer surface as topographic contrast, yielding welldefined structures (see Figure $4 \mathrm{e}$ and cut below). The period and 

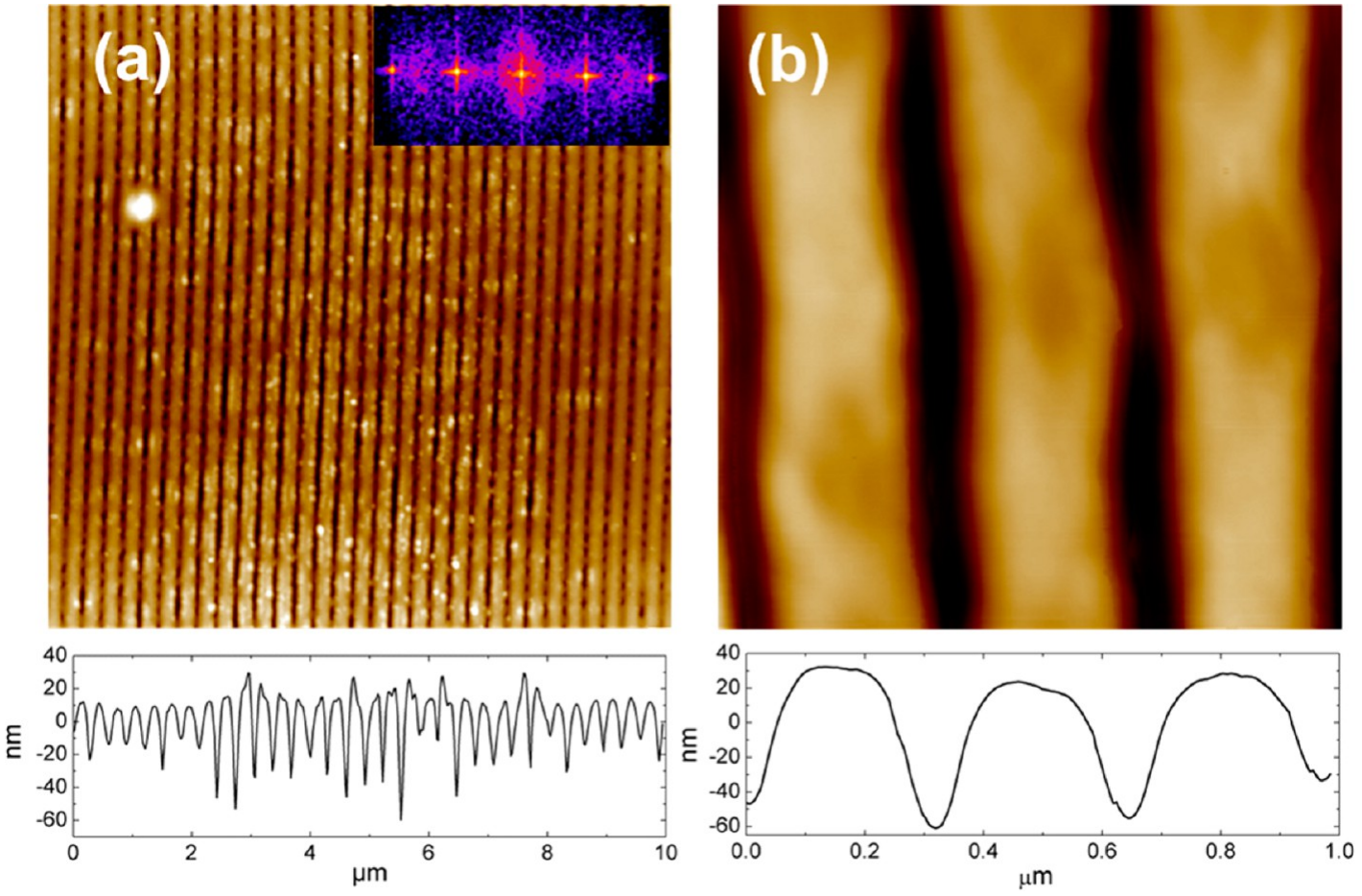

Figure 5. Submicrometer structures generated in a PTT thin film by 2-beam SPLIL (angle of incidence $\theta_{2}=25^{0}$ ): AFM topography maps of patterned areas of (a) $10 \times 10 \mu \mathrm{m}^{2}$ and (b) $1 \times 1 \mu \mathrm{m}^{2}$. Below, height profiles along a line perpendicular to the main axis of the structures. The inset in (a) corresponds to the fast Fourier transform of the image shown.
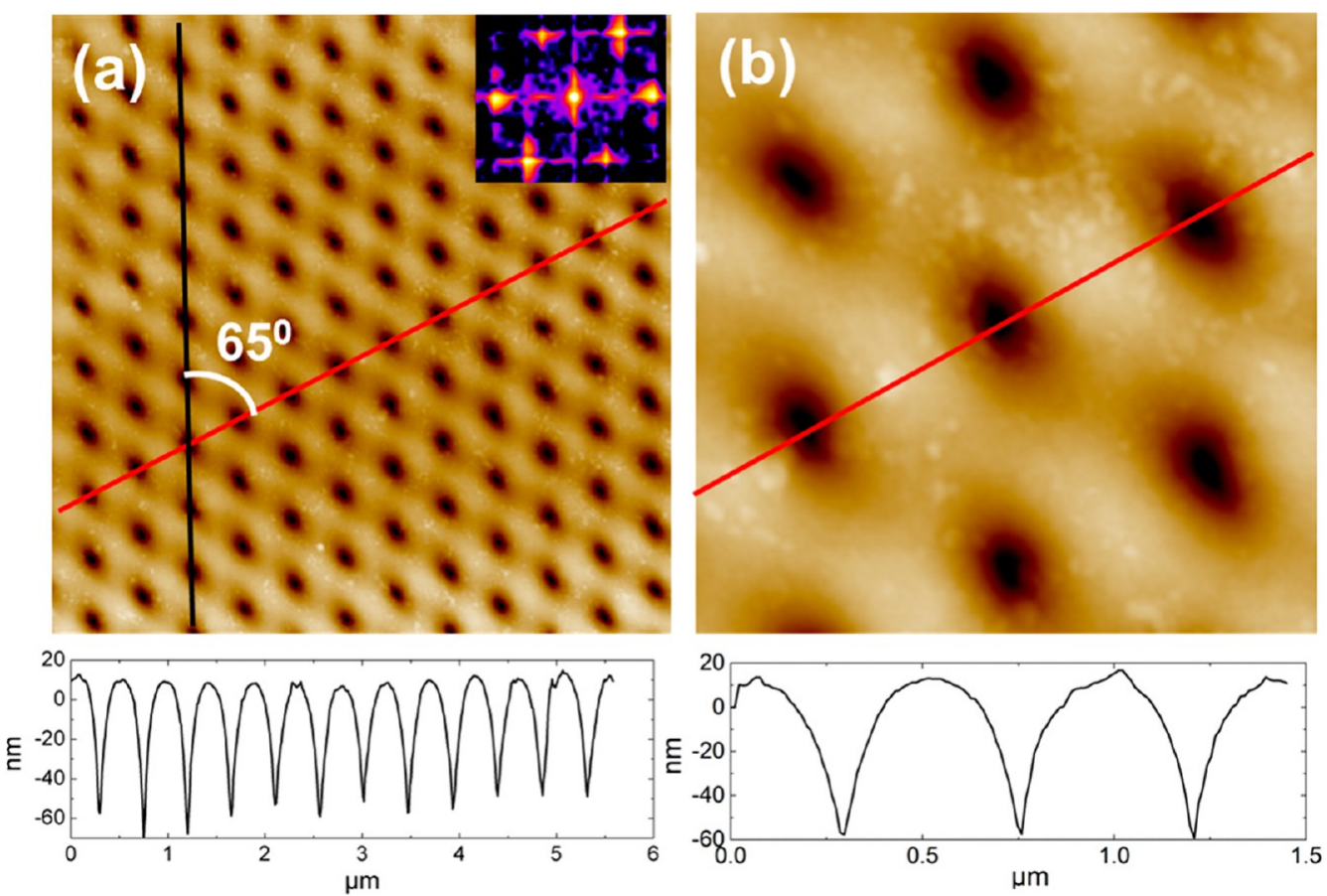

Figure 6. Submicrometric cavities generated in a PTT film by 3-beam SPLIL. AFM topography maps of (a) $10 \times 10 \mu \mathrm{m}^{2}$ and (b) $1.3 \times 1.3 \mu \mathrm{m}^{2}$. Below, height profiles along the red lines depicted, and fast Fourier trasform of the image.

height of the structures as determined by AFM are $\Lambda_{\theta 1}=(1.61 \pm$ 0.02) $\mu \mathrm{m}$ and $\mathrm{H}_{\theta 1}=(195 \pm 13) \mathrm{nm}$, respectively.

Due to the Gaussian shape of the laser beam, the intensity dependence of the structuring process can be studied by examining the pattern evolution when moving from outside the irradiated region toward the center of the laser spot. Figure 4 shows AFM images taken at different areas of the sample.
The morphology obtained outside the irradiated region (Figure 4a) corresponds to the expected one for a spin-coated PTT thin film, extremely flat. Inside the irradiated zone, but close to its edge ( $3.2 \mathrm{~mm}$ from the beam center), slight signs of irradiation can be observed, indicating that the process is initiated by linear absorption (Figure 4b). This leads to gentle ablation without thermal effects near the threshold. The rugosity of the 

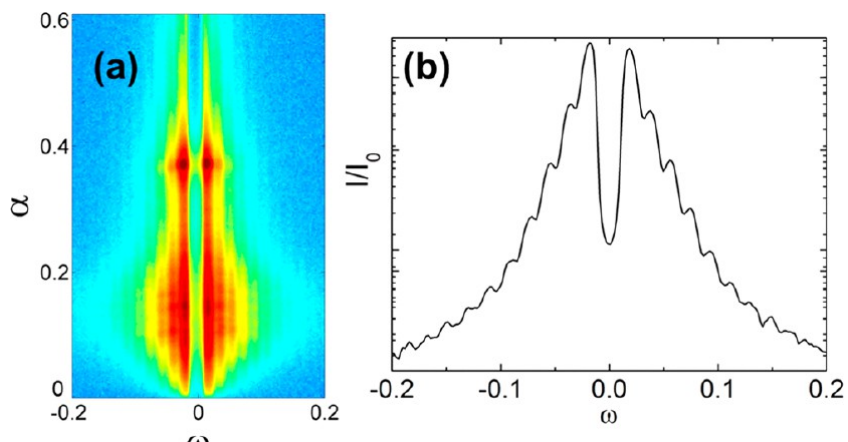

(1)

Figure 7. GISAXS diagram of the sample structured by 3-beam laser interference lithography obtained by aligning the $\mathrm{X}$-ray beam direction with the red line depicted in Figure 3b, (b) $\omega$-cut at an exit angle $\alpha_{\mathrm{f}}=0.15^{\circ}$. film increases and the laser interference fringes start to give rise to topographic contrast (see Figure $4 \mathrm{~b}$, below). If one keeps on approaching the beam center, ablation holes appear along the mentioned fringes (Figure 4c, $2.8 \mathrm{~mm}$ from the beam center), providing evidence of a nonlinear response of the material, where above a certain threshold very efficient material removal takes place. This threshold is in our opinion the boiling threshold, which implies that, below this threshold (in the regime in which only shallow ablation is observed), the film is melted. These observations are consistent with the long penetration depth of the light $(385 \mathrm{~nm})$ compared to the film thickness, leading to a homogeneous temperature increase throughout the film. Even closer to the beam center, these ablation holes merge, (Figure 4d, $2.4 \mathrm{~mm}$ from the beam center) generating bigger holes that start to resemble the final result obtained at the region of optimum
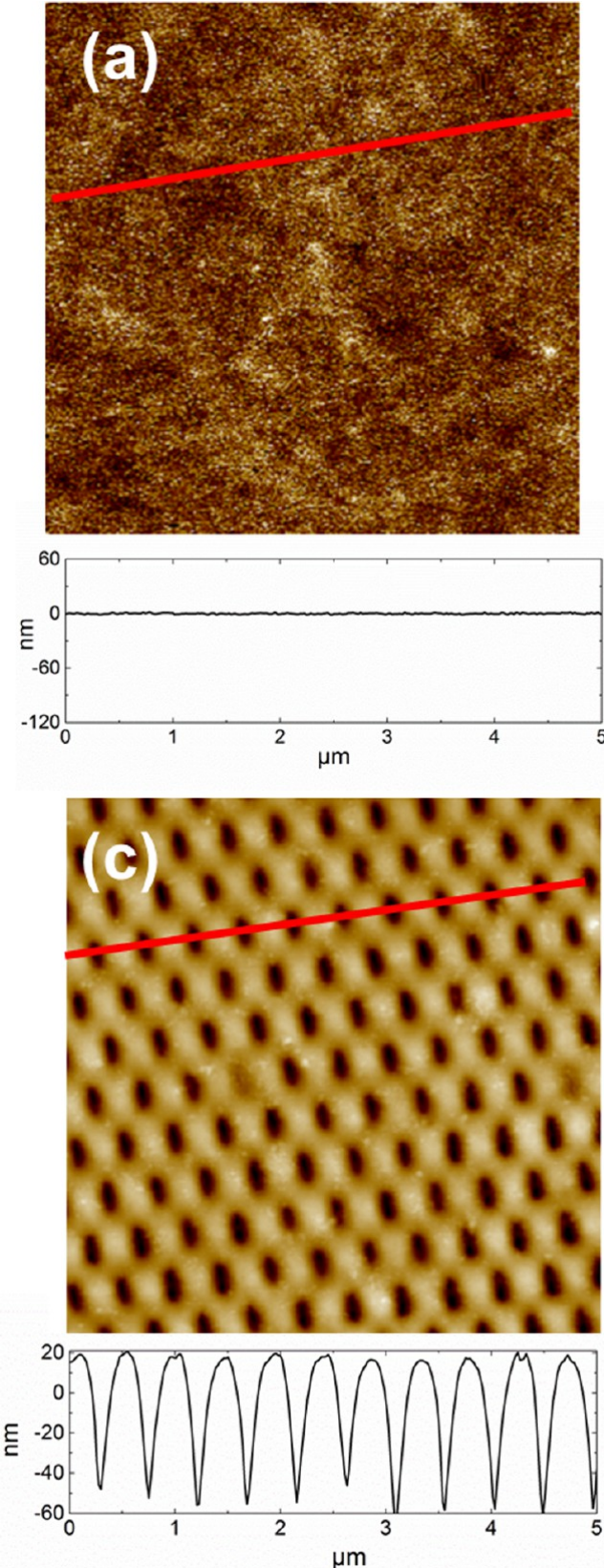
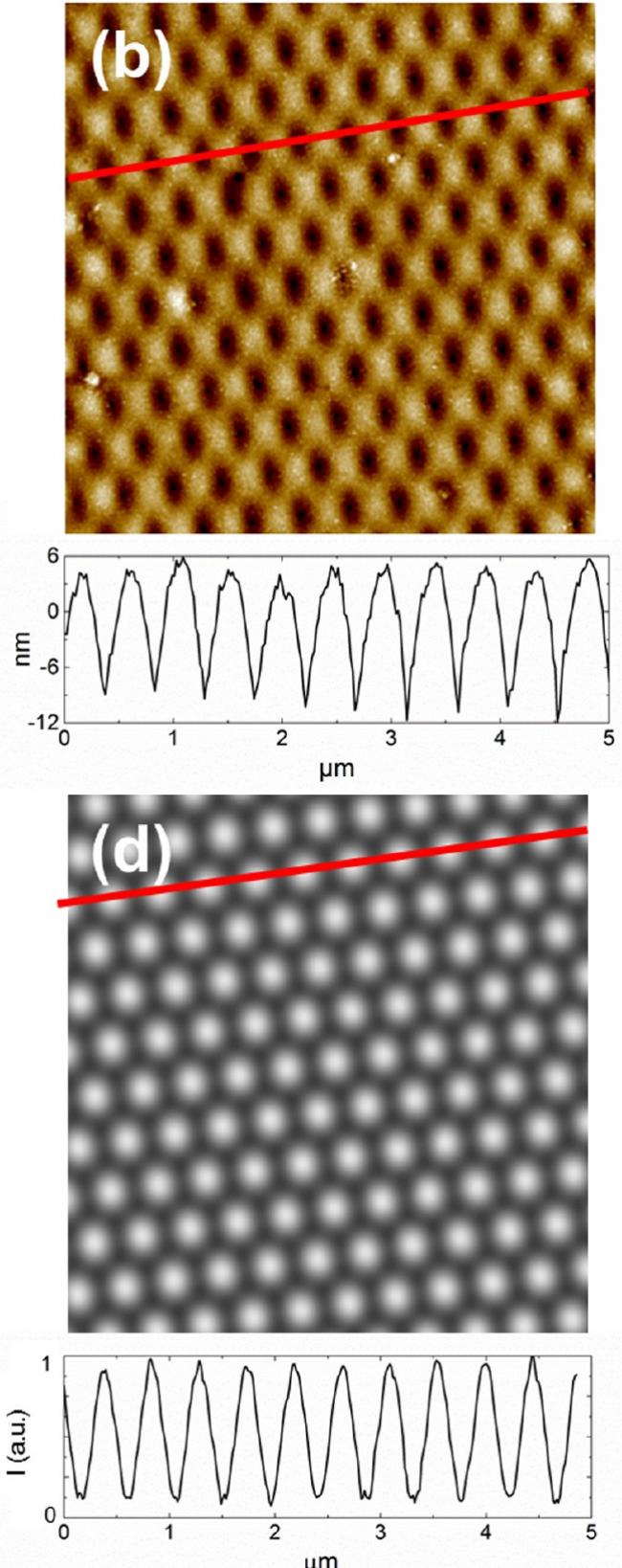

Figure 8. AFM topography images $\left(5 \times 5 \mu \mathrm{m}^{2}\right)$ of submicrometric cavities generated in a PTT film by three-beam single pulse laser interference taken at different areas of the sample: (a) outside the irradiated area and at two different regions inside the irradiated area in descending order of the distance to the beam center: (b) $2.8 \mathrm{~mm}$ and (c) $2 \mathrm{~mm}$. (d) Theoretical interference profile considering the geometry of the experiment. Below, profiles along the red lines depicted. 
fluence close to the spot center (Fig. 4e, $2 \mathrm{~mm}$ from the beam center). The effects of the ablation pressure in the structuring process are revealed in the elevated rims that surround the trenches (cut below Figure 4e), that prove the occurrence of lateral material flow. The final result is therefore similar to the inverted profile of that of the theoretical interference profile for the geometry of the experiment, which is a sine wave (Figure 4f).

Increasing the angle of incidence, the period of the structures can be remarkably reduced, making possible the fabrication of submicrometric gratings (see Figure 5 and cuts below). The period and height of the structures as determined by AFM are $\Lambda_{\theta 2}=(312 \pm 10) \mathrm{nm}$ and $H_{\theta 2}=(59 \pm 22) \mathrm{nm}$, respectively. The presence of the second-order in the Fast Fourier Transform (FFT) of the image (inset in Figure 5a) implies that the markspace ratio is different from $1: 1,{ }^{29}$ illustrating that the width of the trenches is different from that of the protrusions.

In the next set of experiments, three-beam single pulse laser interference was applied to PTT thin films. In this case, the incident beam directions were $\alpha=119^{\circ}, \beta=126.5^{\circ}$, and $\gamma=114.5^{0}$. The results are shown in Figure 6. Submicrometric cavities have been generated in the polymer surface, distributed in a hexagonal lattice (Figure 6a), which is slightly distorted due to the fact that $\alpha \neq \beta \neq \gamma$. Indeed, the black and blue lines define a $65^{\circ}$ angle, instead of the $60^{\circ}$ expected for a perfect hexagonal lattice. As it can be seen in the AFM image and height profile shown in Figure 6a, the structures show a well-defined periodicity, being the period along the red line $\Lambda_{\text {red }}=(456 \pm 3) \mathrm{nm}$, and its height $\mathrm{H}_{\mathrm{red}}=(64 \pm 27) \mathrm{nm}$. Along the black line, however, the periodicity is larger, $\Lambda_{\text {black }}=497 \pm 4 \mathrm{~nm}$. These two periodicities correspond to the parameters of the unit cell of the distorted hexagonal lattice. The shape asymmetry of the cavities (Figure 6b) is most likely caused by slight intensity differences in the local fluence.

GISAXS experiments were performed on the structures prepared by three-beam interference lithography, in order to assess the average structural order of the samples. The sample was arranged in such a way that the X-ray beam was aligned parallel to the red line depicted in Figure $6 \mathrm{~b}$, obtaining the GISAXS diagram shown in Figure 7a. It features scattering maxima out of the meridian $(\omega \neq 0)$, indicating the presence of a well-defined periodicity in the direction perpendicular to the red line (X-ray beam direction). To further analyze this periodicity, a cut at an exit angle of $\alpha_{\mathrm{f}}=0.15^{\circ}$, close to the critical angle of the polymer, is represented in Figure $7 \mathrm{~b}$. In a first approach, the period $\Lambda$ of the nanostructures can be determined through the expression $\Lambda=2 \pi / \mathrm{q}_{y}{ }^{\max }$, where $\mathrm{q}_{y}{ }^{\max }$ is the reciprocal scattering vector corresponding to the first intensity maximum next to $\omega=0 .{ }^{30}$ However, a more accurate value for the period can be obtained by averaging the spacings derived from outer maxima, consecutive orders of the first one, which in our case appears too close to the beamstop. The value obtained this way is $\Lambda_{3 \text { beam }}^{\text {GISAXS }}=$ $431 \pm 3 \mathrm{~nm}$. This figure agrees with the distance between two consecutive and parallel red lines in Fig. 6 a as measured by AFM, which is $\Lambda_{3 \text { beam }}^{\mathrm{AFM}}=(432 \pm 5) \mathrm{nm}$. Assuming an hexagonal lattice of parameter $a$, this can be derived from the observed spacing $D$ value according to the expression $a=2 D / \sqrt{3}$. Then $a=$ $(497 \pm 3) \mathrm{nm}$, in agreement with the lattice parameter measured along the black line of Fig 6a. Thus, AFM and GISAXS agree and provide complementary information, in real and reciprocal space.

The Gaussian shape of the laser beam is again reflected in the morphology of the structures formed. Figure 8 shows AFM images taken at different areas of the sample. The morphology obtained outside the irradiated region (Figure 8a) corresponds to the expected one for a spin-coated PTT thin film, extremely flat. Inside the irradiated zone, but somehow far from the beam center (Figure $8 \mathrm{~b}, 2.8 \mathrm{~mm}$ ), gentle ablation is present and cavities are formed, but they are shallow, with heights no larger than $10-15 \mathrm{~nm}$. However, closer to the beam center (Figure $8 \mathrm{c}, 2 \mathrm{~mm}$ from it), the cavities are deeper, reaching depths of $80-100 \mathrm{~nm}$. The final result is therefore similar to the inverted profile of that of the theoretical interference profile for the geometry of the experiment (Figure 8d).

\section{CONCLUSIONS}

Two- and three-beam SPLIL has been applied to thin films of PTT, obtaining well-defined micro and submicrogratings as well as submicrometer cavities arranged in a distorted hexagonal lattice. The assessment of the resulting structures has been carried out in real (AFM and SEM) and reciprocal (GISAXS) space, showing the solid structural order of the samples and the well-defined morphology of the structures. The formation mechanism of ablation in polymer thin films has been pictured by inspecting different regions of the sample corresponding to different laser fluences, establishing three different regimes in the process. The results highlight the enormous potential of SPLIL as a simple and efficient means for homogeneously micro and submicrostructuring polymer thin films for applications in diverse fields, such as photovoltaics, sensing, or nonvolatile memories.

\section{AUTHOR INFORMATION}

\section{Corresponding Author}

*E-mail: i.fabiani@csic.es.

\section{Notes}

The authors declare no competing financial interest.

\section{ACKNOWLEDGMENTS}

The authors acknowledge financial support from the MINECO (grants MAT2011-23455, MAT2012-33517, TEC2011-22422 and FPI BES-2010-030074). We thank A. Szymczyk and Z. Rosłaniec for providing PTT samples.

\section{REFERENCES}

(1) Hu, J. Q.; Bando, Y.; Liu, Q. L.; Golberg, D. Laser-ablation growth and optical properties of wide and long single-crystal $\mathrm{SnO}_{2}$ ribbons. $A d v$. Funct. Mater. 2003, 13, 493-496.

(2) Gattass, R. R.; Mazur, E. Femtosecond laser micromachining in transparent materials. Nat. Photonics 2008, 2, 219-225.

(3) David, C.; Wei, J.; Lippert, T.; Wokaun, A. Diffractive grey-tone phase masks for laser ablation lithography. Microelectron. Eng. 2001, 57$8,453-460$.

(4) Wolferen, H. V.; Abelmann, L. Laser interference lithography. In Lithography: Principles, Processes and Materials; Hennessy, T. C., Ed; Nova Science Publishers, Inc.: New York, 2011; pp 133-148.

(5) de Boor, J.; Geyer, N.; Goesele, U.; Schmidt, V. Three-beam interference lithography: Upgrading a Lloyd's interferometer for singleexposure hexagonal patterning. Opt. Lett. 2009, 34, 1783-1785.

(6) Xia, L.; Wei, Z. X.; Wan, M. X. Conducting polymer nanostructures and their application in biosensors. J. Colloid Interface Sci. 2010, 341, $1-11$.

(7) Chen, D.; Zhao, W.; Russell, T. P. P3HT nanopillars for organic photovoltaic devices nanoimprinted by AAO templates. ACS Nano 2012, 6, 1479-1485.

(8) Hu, Z. J.; Tian, M. W.; Nysten, B.; Jonas, A. M. Regular arrays of highly ordered ferroelectric polymer nanostructures for non-volatile low-voltage memories. Nat. Mater. 2009, 8, 62-67. 
(9) Martinez-Tong, D. E.; Soccio, M.; Garcia-Gutierrez, M. C.; Nogales, A.; Rueda, D. R.; Alayo, N.; Perez-Murano, F.; Ezquerra, T. A. Improving information density in ferroelectric polymer films by using nanoimprinted gratings. Appl. Phys. Lett. 2013, 102, 191601.1-5.

(10) Pyda, M.; Boller, A.; Grebowicz, J.; Chuah, H.; Lebedev, B. V.; Wunderlich, B. Heat capacity of poly(trimethylene terephthalate). J. Polym. Sci., Part B: Polym. Phys. 1998, 36, 2499-2511.

(11) Lewin, M. Handbook of Fiber Chemistry, 3rd ed.; CRC Press: Boca Raton, FL, 2007.

(12) Wang, Y. Q.; Zhu, H.; Li, B. J. Optical characterization of mechanically tunable microwire based resonators by changing ring radius and wire diameter. Opt. Commun. 2011, 284, 3276-3279.

(13) Xing, X.; Zhu, H.; Wang, Y.; Li, B. Ultracompact photonic coupling splitters twisted by PTT nanowires. Nano Lett. 2008, 8, 28392843.

(14) Yu, H.; Liao, D.; Johnston, M. B.; Li, B. All-optical full-color displays using polymer nanofibers. ACS Nano 2011, 5, 2020-2025.

(15) Martin-Fabiani, I.; Rebollar, E.; Perez, S.; Rueda, D. R.; GarciaGutierrez, M. C.; Szymczyk, A.; Roslaniec, Z.; Castillejo, M.; Ezquerra, T. A. Laser-induced periodic surface structures nanofabricated on poly(trimethylene terephthalate) spin-coated films. Langmuir 2012, 28, $7938-7945$.

(16) Rueda, D. R.; Martin-Fabiani, I.; Soccio, M.; Alayo, N.; PerezMurano, F.; Rebollar, E.; Garcia-Gutierrez, M. C.; Castillejo, M.; Ezquerra, T. A. Grazing-incidence small-angle X-ray scattering of soft and hard nanofabricated gratings. J. Appl. Crystallogr. 2012, 45, 10381045 .

(17) Martin-Fabiani, I.; Siegel, J.; Riedel, S.; Boneberg, J.; Ezquerra, T. A.; Nogales, A. Nanostructuring thin polymer films with optical near fields. ACS Appl. Mater. Interfaces 2013, 5, 11402-11408.

(18) Müller-Meskamp, L.; Kim, Y. H.; Roch, T.; Hofmann, S.; Scholz, R.; Eckardt, S.; Leo, K.; Lasagni, A. F. Efficiency enhancement of organic solar cells by fabricating periodic surface textures using direct laser interference patterning. Adv. Mater. 2012, 24, 906-910.

(19) Perez-Hernandez, H.; Lasagni, A. F. Fast and efficient manufacturing method of one- and two-dimensional polyethylene terephthalate transmission diffraction gratings by direct laser interference patterning. Polym. Eng. Sci. 2012, 52, 1903-1908.

(20) Lasagni, A. F.; Shao, P.; Hendricks, J. L.; Shaw, C. M.; Martin, D. C.; Das, S. Direct fabrication of periodic patterns with hierarchical subwavelength structures on poly(3,4-ethylene dioxythiophene)-poly(styrene sulfonate) thin films using femtosecond laser interference patterning. Appl. Surf. Sci. 2010, 256, 1708-1713.

(21) Lasagni, A. F.; Hendricks, J. L.; Shaw, C. M.; Yuan, D.; Martin, D. C.; Das, S. Direct laser interference patterning of poly(3,4-ethylene dioxythiophene)-poly(styrene sulfonate) (PEDOT-PSS) thin films. Appl. Surf. Sci. 2009, 255, 9186-9192.

(22) Frank, P.; Shaw-Stewart, J.; Lippert, T.; Boneberg, J.; Leiderer, P. Laser-induced ablation dynamics and flight of thin polymer films. Appl. Phys. A: Mater. Sci. Process 2011, 104, 579-582.

(23) Acevedo, D. F.; Lasagni, A. F.; Cornejo, M.; Politano, M.; Barbero, C.; Muecklich, F. Large area fabrication of tuned polystyrene/ poly(methylmethacrylate) periodic structures using laser interference patterning. Langmuir 2009, 25, 9624-9628.

(24) Broglia, M. F.; Suarez, S.; Soldera, F.; Mucklich, F.; Barbero, C. A.; Bellingeri, R.; Alustiza, F.; Acevedo, D. Direct laser interference patterning of polystyrene films doped with azo dyes, using $355 \mathrm{~nm}$ laser light. Appl. Surf. Sci. 2014, 300, 86-90.

(25) Szymezyk, A.; Senderek, E.; Nastalczyk, J.; Roslaniec, Z. New multiblock poly(ether-ester)s based on poly(trimethylene terephthalate) as rigid segments. Eur. Polym. J. 2008, 44, 436-443.

(26) Perlich, J.; Rubeck, J.; Botta, S.; Gehrke, R.; Roth, S. V.; Ruderer, M. A.; Prams, S. M.; Rawolle, M.; Zhong, Q.; Koerstgens, V.; MuellerBuschbaum, P. Grazing incidence wide angle X-ray scattering at the wiggler beamline BW4 of HASYLAB. Rev. Sci. Instrum. 2010, 81, 105105.1-7.

(27) Muller-Buschbaum, P. Grazing incidence small-angle X-ray scattering: An advanced scattering technique for the investigation of nanostructured polymer films. Anal. Bioanal. Chem. 2003, 376, 3-10.
(28) Ezquerra, T. A.; García-Gutiérrez, M. C.; Nogales, A.; Gómez, M., Ed. Applications of Synchrotron Light to Scattering and Diffraction in Materials and Life Sciences; Springer: Berlin, 2009.

(29) Lewis, M. F.; West, C. L. Weighted optical diffraction gratings. Appl. Opt. 1988, 27, 2357-2362.

(30) Rebollar, E.; Perez, S.; Hernandez, J. J.; Martin-Fabiani, I.; Rueda, D. R.; Ezquerra, T. A.; Castillejo, M. Assessment and formation mechanism of laser-induced periodic surface structures on polymer spincoated films in real and reciprocal space. Langmuir 2011, 27, 55965606. 